\title{
Eficiencia energética de la producción de destetes en vacas Brahman (Bos indicus) y cruzadas (Bos taurus x Bos indicus) en Yucatán, México
}

\author{
Cárdenas, M.J.V. ${ }^{1} ;$ Kú, V.J.C. ${ }^{2}$ y Magaña, M.J.G. ${ }^{2}$
}

\begin{abstract}
'Instituto Tecnológico de Tizimín. Departamento de Posgrado e Investigación. Tizimín. Yucatán. México.
2Universidad Autónoma de Yucatán. Facultad de Medicina Veterinaria y Zootecnia. Departamento de Nutrición Animal. Yucatán, México.
\end{abstract}

Palabras ClaVe adicionales

Vacas.

Genotipo.

Destete.

Eficiencia energética.

\section{AdDITIONAL KEYWORDS}

Cows.

Genotype.

Weaning.

Energy efficiency.

\section{INFORMACIÓN}

\section{Cronología del artículo.}

Recibido/Received: 28.3.2014

Aceptado/Accepted: 11.3.2015

On-line: 10.6 .2015

Correspondencia a los autores/Contact e-mail:

valcarme@hotmail.com

\section{INTRODUCCIÓN}

En el trópico mexicano, el ganado Bos indicus representa la mayor proporción de la población bovina, y con el objetivo de incrementar la productividad, han sido introducidas razas $B$. taurus, resultando en la conformación de nuevos genotipos. Para incrementar la productividad y rentabilidad de la ganadería tropical

\section{RESUMEN}

El efecto del genotipo de vaca fue evaluado sobre la eficiencia energética de la producción de destetes (EEPD), en tres grupos de vacas: 15 Brahman (Bos indicus) y 44 cruzadas (B. taurus $\times$ B. indicus) (11 Charoláis $\times$ Brahman y 33 Suizo Pardo $\times$ Brahman), criando becerros de sementales Brahman (Bos indicus), en condiciones de pastoreo extensivas en Yucatán, México. Se obtuvieron datos productivos de las vacas y sus becerros, se calculó el gasto energético de cada estado fisiológico y se estimó la eficiencia energética de la producción de destetes (gramos [g] de destete producidos por megacaloría [Mcal] de energía metabolizable [EM] consumidos por la vaca y el becerro). Existieron diferencias significativas $(p<0,05)$ en la ganancia de peso $(881 \pm 190$ y $726 \pm 165 \mathrm{~g} / \mathrm{d}$ para becerros cruzados y Brahman respectivamente) y peso al destete $(200 \pm 35$ y $166 \pm 27 \mathrm{~kg}$ para becerros cruzados y Brahman respectivamente) de los becerros, por efecto del genotipo de la vaca. Las vacas cruzadas $(B$. taurus $\times B$. indicus) tuvieron $13,1 \%(p<0,05)$ más EEPD con respecto a las vacas Brahman $(19,8 \pm 2,8$ vs. 17,5 $\pm 2,7 \mathrm{~g} /$ Mcal de EM para vacas cruzadas y Brahman respectivamente). En Yucatán, México, se puede incrementar la producción de destetes por ciclo, utilizando vacas cruzadas (B. taurus $\times$ B. indicus) en condiciones de pastoreo.

\section{Energy efficiency of weaning production in Brahman (Bos indicus) and crossed (Bos taurus x Bos indicus) cows in Yucatan, Mexico}

\section{SUMMARY}

Cow genotype effect was evaluated in energy efficiency of weaning production (EEWP), on three cow groups: 15 Brahman (Bos indicus) and 44 crossed cows (B. taurus $\times$ B. indicus) (11 Charolais $\times$ Brahman and 33 Brown Swiss $\times$ Brahman), breeding Brahman sire calves, under grazing extensive conditions in Yucatan, Mexico. Productive data of cows and calves was obtained, energetic expenses were calculated for every physiologic state and energy efficiency of weaning production was estimate (gram [g] of weaning weight produced per mega calorie [Mcal] of metabolizable energy $[\mathrm{ME}]$ consumed by cow and calf). There were statistic differences $(p<0.05)$ in weight gain $(881 \pm 190$ and $726 \pm 165 \mathrm{~g} / \mathrm{d}$ for calves of crossed and Brahman cows) and weaning weight $(200 \pm 35$ and $166 \pm 27 \mathrm{~kg}$ for calves of crossed and Brahman cows) of calves, despite cow genotype effect. Crossbred cows (B. taurus $\times B$. indicus) had $13.1 \%(p<0.05)$ more EEWP than Brahman cows $(19.8 \pm 2.8 \mathrm{vs} .17 .5 \pm 2.7 \mathrm{~g} /$ Mcal of EM for crossbred and Brahman cows). In Yucatan, Mexico, it is possible increase weaning production in a cycle, by the use of crossbred cows (B. taurus $\times B$. indicus) under grazing conditions. es necesario compatibilizar los genotipos disponibles con el ambiente.

Las razas bovinas tienen diferentes atributos, como adaptación al ambiente tropical en el Brahman (Bos indicus), y producción de leche, habilidad materna y potencial de crecimiento en las razas Bos taurus (Francisco et al., 2012). En Yucatán, México, la Brahman (B. indicus) es mayormente utilizada como raza materna, 
en cruzas con sementales B. taurus, generalmente Suizo pardo y Charolais, para obtener vacas F1.

En las cruzas $B$. taurus $\times B$. indicus se ha observado efecto de heterosis para el incremento de peso en la progenie (Aban et al., 2008); sin embargo, al incrementar la proporción de $B$. taurus en vacas en condiciones alimenticias limitadas, disminuye su comportamiento productivo y reproductivo (Green et al., 1991a), debido en gran parte al incremento de sus requerimientos nutricionales (Ferrell y Jenkins, 1984ab).

La eficiencia energética en la producción de destetes es un indicador del balance entre la productividad y la utilización de la energía disponible del alimento (Johnson et al., 2003), esta eficiencia puede variar del 10 al $30 \%$ por diferencias genéticas (Archer et al., 1999), lo que puede significar una oportunidad para aprovechar en el trópico las ventajas de integrar los genotipos al ambiente.

El objetivo del presente estudio fue estimar la eficiencia energética de la producción de destetes en vacas Brahman (B. indicus) y cruzadas (B. taurus $x$ B. indicus), criando becerros de sementales Brahman ( $B$. indicus) en Yucatán, México.

\section{MATERIAL Y MÉTODOS}

Los datos para el estudio fueron recabados en un sistema de producción de destetes del municipio de Panabá, en el estado de Yucatán, México, localizado entre $19^{\circ} 40^{\prime}$ norte y $87^{\circ} 32^{\prime}$ oeste, con clima cálido subhúmedo, con lluvias de junio a octubre, y una temperatura anual de 24,5 a $27,5^{\circ} \mathrm{C}$ (Duch, 1988).

Para estimar la eficiencia energética de la producción de destetes se obtuvieron datos productivos de vacas y sus becerros en un ciclo de producción, y se utilizaron para calcular el gasto energético de cada estado fisiológico.

Se realizó un seguimiento del comportamiento de 59 vacas multíparas de 7 a 9 años de edad, pertenecientes a tres genotipos: 15 Brahman (B. indicus), 33 Suizo Pardo (B. taurus) x Brahman (F1) y 11 Charoláis (B. taurus) $x$ Brahman (F1), criando becerros de sementales Brahman (B. indicus). El registro de datos inició durante el mes de abril, las vacas y becerros fueron identificados y pesados durante las primeras 12 horas posparto, los becerros se criaron en amamantamiento continuo hasta el destete.

El manejo de los animales consistió en pastoreo de 17:00 a 06:00 horas, en potreros de Guinea (Panicum maximum) a temporal, con carga animal promedio de una unidad animal por hectárea. Durante el día, los animales se mantuvieron en corral para el consumo de agua, sales minerales y cuidados en general. Durante el ciclo de producción las vacas recibieron alimentación de apoyo con pasto Taiwán molido (Pennisetum purpureum).

El peso vivo $(\mathrm{kg})$ de vacas y crías se registró al parto, cada dos meses hasta el destete, y posteriormente el peso de las vacas al siguiente parto. El pesaje se llevó a cabo durante la mañana, con retiro de alimento y agua 12 horas previas. El peso de las vacas se utilizó para determinar los cambios de peso vivo (CPV) en el ciclo de producción. El peso de los becerros se utilizó para estimar la ganancia de peso predestete (GDP), y por regresión se obtuvo el peso de la cría a los 180 días, edad promedio a la cual se realiza el destete en Yucatán, lo cual fue considerado como peso al destete (PD).

La producción de leche total (PLT) por vaca fue determinada cada dos meses posparto hasta el destete. La cría se retiró 12 horas y se aplicaron a la vaca $10 \mathrm{ml}$ de oxitocina por vía intramuscular, se ordeñó manualmente hasta secar completamente la ubre, seis horas después se repitió el proceso para una segunda ordeña, el peso en $\mathrm{kg}$ de la leche obtenido se multiplicó por cuatro para estimar la cantidad de leche producida en 24 horas, la producción de leche total (PLT) se estimó aritméticamente con el promedio de producción (Jenkins y Ferrell, 1992).

El gasto energético de los diferentes estados fisiológicos se estimó utilizando el promedio del peso vivo de los animales en cada etapa, utilizando ecuaciones y coeficientes de los requerimientos de energía metabolizable (EM). La eficiencia energética de la producción de destetes (EEPD) se definió como los gramos de destete producido por megacaloría (Mcal) de EM total proveniente del alimento en un ciclo de producción de destete (g/Mcal de $\mathrm{EM}_{\mathrm{T}}$ ), considerando como energía metabolizable total $\left(\mathrm{EM}_{\mathrm{T}}\right)$ la suma de la energía utilizada para los procesos de mantenimiento de la vaca $\left(\mathrm{EM}_{\mathrm{mv}}\right)$, producción de leche $\left(\mathrm{EM}_{\mathrm{pl}}\right)$, cambios de peso vivo $\left(E M_{\mathrm{cpv}}\right)$, gestación $\left(\mathrm{EM}_{\mathrm{g}}\right)$ y la cría $\left(\mathrm{EM}_{\mathrm{mc}}\right)$.

La EM requerida para las funciones de mantenimiento de la vaca $\left(\mathrm{EM}_{\mathrm{mv}}\right)$, se estimó para cada período de 60 días durante la etapa de crianza y del destete hasta el siguiente parto, utilizando la siguiente ecuación (Montaño y Nielsen, 1990; Green et al., 1991b):

$$
\mathrm{EM}_{\mathrm{mv}}=\sum_{\mathrm{i}=1}^{\mathrm{IEP}}\left\{[\mathrm{PI}+(\mathrm{CPV} \times \mathrm{i})]^{0,75} \times \mathrm{EM}_{\mathrm{m}}\right\}
$$

Donde:

$\mathrm{EM}_{\mathrm{mv}}=$ Mcal de EM para mantenimiento de la vaca,

$\mathrm{IEP}=$ días de intervalo entre partos,

$\mathrm{PI}=$ peso vivo de la vaca al inicio del período $(\mathrm{kg})$,

i= día en el ciclo de producción de destete,

$\mathrm{CPV}=$ cambio de peso vivo de la vaca $(\mathrm{kg})$ en el ciclo de producción de destete,

$\mathrm{EM}_{\mathrm{m}}=\mathrm{EM}$ para mantenimiento de la vaca por $\mathrm{kg}$ de peso metabólico (kcal EM/ $\mathrm{kg}^{0,75} / \mathrm{d}$ ).

El requerimiento de EM para la producción de leche $\left(\mathrm{EM}_{\mathrm{pl}}\right)$ se estimó utilizando la PLT por vaca, asumiendo 1.06 Mcal de EM por $\mathrm{kg}$ de PLT, lo cual corresponde a una eficiencia de utilización de la EM para lactación de $\left(k_{1}\right)$ 0,70, mediante la siguiente ecuación (NRC, 2000):

$$
\mathrm{EM}_{\mathrm{pl}}=\mathrm{PLT} * 1,06
$$

\section{Donde:}

$\mathrm{EM}_{\mathrm{pl}}=$ Mcal de EM requeridos para lactación, y

PLT $=$ Producción de leche total $(\mathrm{kg})$. 
El requerimiento de EM durante la gestación $\left(\mathrm{EM}_{\mathrm{g}}\right)$ contiene los componentes de mantenimiento y gestación, los cuales son determinados para los 60 días previos al parto, utilizando la siguiente ecuación (NRC, 2000):

$$
\mathrm{EM}_{\mathrm{g}}=\left(\mathrm{p}^{0,75} * 173\right) * 60
$$

Donde:

$\mathrm{EM}_{\mathrm{g}}=$ Mcal de EM para mantenimiento y gestación durante 60 días previos al parto,

$\mathrm{P}^{0,75}=$ peso metabólico promedio a 60 días previos al parto;

$173=\mathrm{kcal}$ de $\mathrm{EM} / \mathrm{Kg}^{0,75}$ por día;

$60=$ días previos al parto.

El peso de la vaca 60 días al parto $(\mathrm{kg})$ se estimó por regresión del peso al destete y del siguiente parto. La $\mathrm{EM}_{\mathrm{g}}$ se calculó asumiendo una eficiencia de uso de la $\mathrm{EM}^{\mathrm{g}}$ del alimento para la gestación $\left(k_{\mathrm{c}}\right)$ de 0,14 (Green et al., 1991b).

Para estimar los requerimientos de EM de mantenimiento y crecimiento de la cría $\left(\mathrm{EM}_{\mathrm{mc}}\right)$, se utilizó la siguiente ecuación (Montaño y Nielsen, 1990, Green et al., 1991b):

$$
\mathrm{EM}_{\mathrm{mc}}=\sum_{\mathrm{i}=1}^{180}\left\{[\mathrm{PN}+(\mathrm{GDP} \times \mathrm{i})]^{0,75} \times\left[\mathrm{EM}_{\mathrm{m}}+(\mathrm{I} \times \mathrm{i})\right]\right\}
$$

\section{Donde:}

$\mathrm{EM}_{\mathrm{mc}}=$ Mcal de EM para mantenimiento de la cría,

i= día en el ciclo de producción de destete,

$\mathrm{PN}=$ peso de la cría al nacimiento $(\mathrm{kg})$,

$\mathrm{GDP}=$ ganancia diaria de peso en el período predestete $(\mathrm{kg})$,

$\mathrm{EM}_{\mathrm{m}}=\mathrm{EM}$ requerida para mantenimiento de la cría por unidad de peso metabólico (kcal de EM/ $\left.\mathrm{kg}^{0,75} / \mathrm{d}-1\right)$,

I= Incremento diario del requerimiento de EMm por concepto de crecimiento ( $\mathrm{kcal}$ de $\mathrm{EM} / \mathrm{kg}^{0,75} / \mathrm{d}-1$ ).

El coeficiente para $\mathrm{EM}_{\mathrm{m}}$ utilizado fue de 113,4 y $119,5 \mathrm{kcal} / \mathrm{kg}^{0,75} / \mathrm{d}$ para vacas Brahman y cruzadas respectivamente (Cárdenas et al., 2010). Los valores de $\mathrm{EM}_{\mathrm{m}}$ y crecimiento (I) para la progenie fueron los mismos que para las vacas Brahman $\left(113,4 \mathrm{kcal} / \mathrm{kg}^{0,75} / \mathrm{d}\right)$, debido a que la progenie es resultado de la cruza $B$. indicus $\times$ B. indicus, y de la retrocruza $B$. indicus x (B. taurus $\times$ B. indicus).
Para ajustar los requerimientos de $\mathrm{EM}_{\mathrm{m}}$ por los cambios de peso vivo de la vaca del parto al destete $\left(E M_{\text {cpv }}\right)$, y considerando un contenido de 7,11 Mcal de $\mathrm{EM}$ por $\mathrm{kg}$ de peso vivo, al requerimiento de $\mathrm{EM}_{\mathrm{m}}$ de cada período de 60 días se le sumaron 12,5 Mcal de EM por cada $\mathrm{kg}$ de peso vivo ganado (Cárdenas et al., 2010), y 8,5 Mcal de EM por cada $\mathrm{kg}$ de peso perdido (Ferrell y Jenkins, 1984a, Green et al., 1991b), lo que corresponde a una eficiencia del uso de la EM para la ganancia y pérdida de peso corporal de 0,57 y 0,84 respectivamente (Moe y Tyrrell, 1972).

Las variables del comportamiento de vacas y crías, y los requerimientos energéticos para mantenimiento y eficiencia energética de producción de destetes, fueron analizadas por el procedimiento de Modelos Generales Lineales (SAS, 2002), mediante un modelo de efectos fijos. El modelo fijo inicial incluyó el efecto del grupo genético de la vaca, el número de parto, el sexo de la cría y su interacción, pero debido a que la interacción, el número de parto de la vaca y el sexo de la cría no fueron estadísticamente significativos, fueron excluidos del análisis final. Para estimar diferencias dentro de los efectos fijos, se realizó un análisis de comparación de medias utilizando una prueba de rango múltiple de Duncan (Steel y Torrie, 1980).

\section{RESULTADOS Y DISCUSIÓN}

En la tabla I se muestran los resultados del seguimiento dinámico del comportamiento de vacas y sus crías en un ciclo de producción de destete.

El peso promedio de las vacas fue de $498 \pm 60 \mathrm{y}$ $519 \pm 50 \mathrm{~kg}$ al parto y final respectivamente, y no se encontraron diferencias significativas $(p>0.05)$ entre los genotipos de vaca, lo que sugiere la posibilidad que en Yucatán las vacas Brahman (BR) y cruzadas (B. taurus $x$ B. indicus), con similar edad y número de partos presenten similar peso vivo maduro. El peso de la vaca se relaciona con su tamaño a la madurez, y tiene influencia en la eficiencia energética de la producción de destetes (Green et al., 1990ab), un incremento en el tamaño de la vaca puede incrementar el costo energético de mantenimiento (Ferrell y Jenkins, 1985), es necesario por lo tanto procurar genotipos compatibles con los recursos alimenticios disponibles (Sandelin et al., 2002). Es necesario identificar las cruzas, que incrementen la eficiencia en la producción de destetes en Yucatán, sin que se presenten las desventajas del incremento en el peso vivo en las vacas.

Tabla I. Promedios y errores estándar del comportamiento en vacas de tres genotipos y sus crías en Yucatán (Means and standard errors of behavior in three genotypes cows and calves in Yucatan).

\begin{tabular}{lcccccc}
\hline & \multicolumn{3}{c}{ Comportamiento de vacas } & \multicolumn{3}{c}{ Comportamiento de crías } \\
\cline { 2 - 7 } Genotipo & Peso al parto $(\mathrm{kg})$ & Peso final $(\mathrm{kg})$ & PLT $(\mathrm{kg})$ & IEP $(\mathrm{d})$ & GDP $(\mathrm{g} / \mathrm{d})$ & PD $(\mathrm{kg})$ \\
\hline BR & $499 \pm 22$ & $519 \pm 18$ & $3.4^{\mathrm{b}} \pm 0,6$ & $426 \pm 15$ & $726^{\mathrm{b}} \pm 64$ & $166^{\mathrm{b}} \pm 11$ \\
CH x C & $509 \pm 19$ & $523 \pm 16$ & $4.9^{\mathrm{ab}} \pm 0,5$ & $398 \pm 14$ & $808^{\mathrm{ab}} \pm 58$ & $187^{\mathrm{ab} \pm 10}$ \\
SP x C & $493 \pm 14$ & $516 \pm 12$ & $6.0^{\mathrm{a}} \pm 0,6$ & $442 \pm 10$ & $918^{\mathrm{a}} \pm 41$ & $207^{\mathrm{a}} \pm 7$ \\
\hline
\end{tabular}

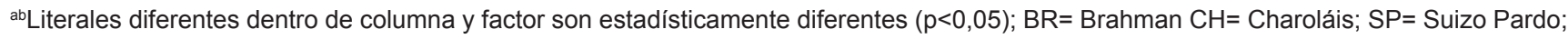
$\mathrm{kg}=$ kilogramos; $\mathrm{PD}=$ peso al destete; $\mathrm{PLT}=$ producción de leche total; IEP= intervalo entre partos; GDP= ganancia diaria de peso. 
La producción de leche total (PLT) en las vacas BR y cruzadas (B. taurus $x$ B. indicus) fue de $3,4 \pm 0,6$ y $5,5 \pm 0,6$ $\mathrm{kg} / \mathrm{d}$ respectivamente, y se detectaron diferencias estadísticas $(p<0,05)$ entre las vacas BR y Suizo Pardo $(\mathrm{SP}) \times \mathrm{BR}$, presentando las vacas Charolais $(\mathrm{CH}) \times \mathrm{BR}$ valores intermedios; las vacas cruzadas produjeron en promedio $60 \%$ más que las vacas BR, estas diferencias están de acuerdo a lo reportado en el trópico (Yilma et al., 2006; Aguilar, 2007; Magaña et al., 2009). El genotipo determina el potencial de producción de leche en vacas, influye en el consumo de EM y la eficiencia energética de la producción de destetes, las vacas con alto potencial de producción de leche tienen órganos grandes con alto costo energético para su funcionamiento, no obstante, es probable que en vacas cruzadas ( $B$. taurus $\times$ B . indicus), con mediano potencial lechero, el costo energético de producción de leche se compense con una mayor ganacia de peso de los becerros (Montaño et al., 1990).

El IEP promedio en las vacas fue de 427,2 $\pm 46,2$ días, y no existieron diferencias significativas $(p>0,05)$ entre los genotipos, de acuerdo con lo obtenido por Mejía et al. (2010), quienes reportan similar comportamiento reproductivo entre vacas cebuinas (B. indicus) y cruzadas (B. taurus x B. indicus) en Yucatán (IEP $=432,9 \pm 96,9$ días). El IEP en las vacas puede influir en la eficiencia energética de la producción de destetes, ya que el consumo total de EM podría aumentar en razón de un incremento de días del ciclo productivo.

Existieron diferencias significativas $(p<0,05)$ en el comportamiento de los becerros debidas al genotipo de la vaca. Los becerros cruzados tuvieron una GDP $21,3 \%$ mayor en comparación de los becerros de vacas BR $(726 \pm 165$ y $881 \pm 190 \mathrm{~g} / \mathrm{d}$ para becerros de vacas BR y cruzadas respectivamente), y fueron en promedio $20,5 \%$ más pesados al destete $(166 \pm 27,1$ y $200 \pm 35$ $\mathrm{kg}$ para becerros BR y cruzados respectivamente). El comportamiento de la cría influye directamente en la eficiencia energética en el sistema vaca:cría, lo cual ha sido reportado por Green et al. (1991a) y Plasse et al. (2000), quienes cuantificaron una variación del 7 a $16 \%$ en el comportamiento del becerro y la eficiencia energética de la producción de destetes, por efecto del genotipo de la vaca. Martínez et al. (2012) por su parte, atribuyeron el comportamiento de los becerros al genotipo de la vaca y su potencial de producción de leche, estimando una correlación significativa $(\mathrm{p}<0,05$, $r=0,44, b=0,049 \pm 0,007)$ entre la producción de leche de vacas (B. indicus, B. taurus x B. indicus) y el peso al destete de los becerros.

En la tabla II se muestran los resultados de los requerimientos de EM para las funciones fisiológicas de las vacas, de las crías, y la eficiencia energética de la producción de destetes.

Las vacas utilizaron en promedio 5506 $4757 \mathrm{y}$ $1374 \pm 755$ Mcal de EM para el mantenimiento $\left(\mathrm{EM}_{\mathrm{mv}}\right)$ y los cambios de peso (EM ${ }_{\mathrm{cpv}}$ ) respectivamente, sin diferencias significativas $(\mathrm{p}>0,05)$ por efecto del genotipo de la vaca, esto se pudo deber probablemente a que entre los genotipos evaluados no existieron diferencias significativas $(p>0,05)$ en los requerimientos de EM para el mantenimiento $\left(\mathrm{EM}_{\mathrm{m}}\right)$, tal como fue evidenciado por Cárdenas et al. (2010), quienes no detectaron diferencias en el EM $\mathrm{m}_{\mathrm{m}}$ entre vacas BR y Suizo Pardo en Yucatán $\left(113 \pm 20\right.$ y $126 \pm 13 \mathrm{kcal} / \mathrm{kg}^{0,75} / \mathrm{d}_{\mathrm{m}} \mathrm{de} \mathrm{EM}_{\mathrm{m}^{\prime}}$ para BR y SP respectivamente), en contraste con Solis et al. (1988) y Calegare et al. (2007), quienes estimaron diferencias $(p<0,05)$ de 20,4 a 33,3\% en el $E M_{m}$ entre vacas cruzadas (B. taurus $\times$ B. indicus) y B. indicus.

Existieron diferencias significativas $(\mathrm{p}<0,05)$ por efecto del genotipo de vaca en la energía utilizada para la producción de leche $\left(\mathrm{EM}_{\mathrm{pl}}\right)$, la energía utilizada por las crías $\left(E M_{m c}\right)$, el gasto energético total $\left(E_{T}\right)$ y la eficiencia energética de la producción de destetes (EEPD). Las vacas cruzadas (B. taurus x B. indicus) utilizaron $60 \%$ más $\mathrm{EM}_{\mathrm{pl}}$ en comparación con las vacas BR $\left(1033 \pm 240\right.$ y $655 \pm 51$ Mcal de $\mathrm{EM}_{\mathrm{pl}}$ para cruzadas y $\mathrm{BR}$ respectivamente), lo cual demuestra el mayor potencial lechero de las vacas cruzadas en comparación con razas $B$. indicus, la raza Suizo Pardo es considerada como de doble propósito y la Charolais como cárnica. Los becerros de vacas cruzadas ( $B$. taurus $\times$ B . indicus) utilizaron un 14,9\% más $\mathrm{EM}_{\mathrm{mc}}$ que los becerros de vacas BR (1088 \pm 141 y $947 \pm 116 \mathrm{Mcal}^{\mathrm{me}} \mathrm{EM}_{\mathrm{mc}}$ para becerros de vacas cruzadas y BR respectivamente). Las vacas cruzadas utilizaron $6,4 \%$ más $\mathrm{EM}_{\mathrm{T}}$ que las vacas BR $\left(10151 \pm 1444\right.$ vs $9543 \pm 458$ Mcal de $\mathrm{EM}_{\mathrm{T}}$ para vacas cruzadas y BR respectivamente). La EEPD fue 13,1\% mayor en vacas cruzadas con respecto a las vacas $B R$ $\left(19,8 \pm 2,8\right.$ vs $17,5 \pm 2,7 \mathrm{~g} / \mathrm{Mcal}$ de $\mathrm{EM}_{\mathrm{T}}$ para vacas cruzadas y BR respectivamente), lo que parece indicar que el incremento en el gasto total de energía $\left(\mathrm{EM}_{\mathrm{T}}\right)$ en las vacas cruzadas (B. taurus $\times$ B. indicus), se compensa por el incremento significativo $(\mathrm{p}<0,05)$ en la eficiencia en la producción de destetes (Archer et al., 1999).

Tabla II. Promedios y errores estándar de los requerimientos de EM y eficiencia energética de la producción de destetes en vacas de tres genotipos en Yucatán (Averages and standard errors of EM requirements and energy efficiency of production in cows weaned three genotypes in Yucatan).

\begin{tabular}{lcccrrr}
\hline Genotipo & $\mathrm{EM}_{\mathrm{mv}}($ Mcal $)$ & $\mathrm{EM}_{\mathrm{cpv}}(\mathrm{Mcal})$ & $\mathrm{EM}_{\mathrm{pl}}($ Mcal $)$ & $\mathrm{EM}_{\mathrm{mc}}($ Mcal $)$ & $\mathrm{EM}_{\mathrm{T}}(\mathrm{Mcal})$ & $\mathrm{EEPD}_{\left(\mathrm{g} / \mathrm{Mcal} \mathrm{EM}_{\mathrm{T}}\right)}$ \\
\hline $\mathrm{BR}$ & $5275 \pm 258$ & $1572 \pm 263$ & $655^{\mathrm{b}} \pm 116$ & $947^{\mathrm{b}} \pm 47$ & $9543^{\mathrm{ab}} \pm 458$ & $17,5^{\mathrm{b}} \pm 1,0$ \\
$\mathrm{CH} \times \mathrm{C}$ & $5198 \pm 203$ & $1032 \pm 236$ & $948^{\mathrm{ab}} \pm 101$ & $1034^{\mathrm{ab}} \pm 42$ & $9332^{\mathrm{b}} \pm 410$ & $20,1^{\mathrm{a}} \pm 0,9$ \\
SPxC & $5751 \pm 163$ & $1467 \pm 167$ & $1147^{\mathrm{a}} \pm 116$ & $1114^{\mathrm{a}} \pm 30$ & $10565^{\mathrm{a}} \pm 290$ & $19,6^{\mathrm{a}} \pm 0,6$ \\
\hline
\end{tabular}

ab Literales diferentes dentro de columna y factor son estadísticamente diferentes $(\mathrm{p}<0,05)$; $\mathrm{BR}=\mathrm{Brahman}$; $\mathrm{CH}=\mathrm{Charoláis;} \mathrm{SP}=\mathrm{Suizo} \mathrm{Pardo}$; $\mathrm{Mcal}=$ megacaloría; $\mathrm{EM}=$ energía metabolizable; $\mathrm{EM}_{\mathrm{mv}}=\mathrm{EM}$ de mantenimiento de la vaca; $\mathrm{EM}_{\mathrm{pl}}=\mathrm{EM}_{\mathrm{Para}}$ la producción de leche; $\mathrm{EM}$ = $\mathrm{EM}$ por los cambios de peso vivo en la vaca; $\mathrm{EM}_{\mathrm{mc}}=\mathrm{EM}$ de mantenimiento y crecimiento de la cría; $\mathrm{EM}_{\mathrm{T}}=\mathrm{EM}_{\mathrm{m}}$ total utilizada por la vaca y su cría; $E E P D=$ eficiencia energética de la producción de destete. 
La eficiencia energética de la producción de destetes (EEPD) promedio $\left(19,3 \pm 2,9 \mathrm{~g} /\right.$ Mcal de $\left.\mathrm{EM}_{\mathrm{T}}\right)$ se encontró por debajo del rango de 33,1 a 47,4 g/Mcal EM, estimado en climas templados (Jenkins et al., 1991; Calegare et al., 2007); sin embargo, de acuerdo con las conclusiones de Davis et al. (1983ab), Jenkins y Ferrell (2004) y Calegare et al. (2009), se pudo constatar un efecto racial $(\mathrm{p}<0,05)$ en la eficiencia de producción de $\mathrm{kg}$ de destete, en relación a la EM proveniente del alimento consumido por las vacas y sus crías.

En Yucatán, las vacas cruzadas F1 (B. taurus x B. indicus) pueden producir más kg de becerro por unidad de EM que proviene de los forrajes, lo que hace posible incrementar la eficiencia por vaca por ciclo productivo, evitar efectos adversos sobre la productividad y reproducción en las vacas, e integrar genotipos $B$. taurus al sistema de producción de destetes; no obstante, es necesario realizar más estudios sobre otros genotipos y sistemas de cruzamientos, ya que existe evidencia que indica que la eficiencia puede verse comprometida, al incrementar la proporción de B. taurus (>50\%) en vacas en condiciones alimenticias limitadas, atribuible a caracteres relacionados con el incremento de los requerimientos nutricionales, como el peso a la madurez y la producción de leche (Ferrell y Jenkins, 1984ab; Montaño y Nielsen, 1990; Green et al., 1991ab; McDowell et al., 1996; Ferrell y Jenkins, 1998a; Fox et al., 2002).

\section{CONCLUSIONES}

En el trópico es necesario utilizar razas o genotipos de vaca para la producción de destetes, con bajo requerimiento de energía para mantenimiento, que puedan sostener niveles de producción y reproducción, sin las desventajes de un alto peso a la madurez. En Yucatán la eficiencia energética de la producción de destetes es mayor en vacas cruzadas F1 (B. taurus x B. indicus) en relación a vacas Brahman (B. indicus), lo que representa una oportunidad para la conformación de genotipos que incrementen la productividad y rentabilidad.

\section{AGRADECIMIENTOS}

Al Consejo Nacional de Ciencia y Tecnología (CONACYT), por el financiamiento otorgado para la realización de los estudios de doctorado del autor principal.

\section{BIBLIOGRAFÍA}

Abán, J.A.; Delgado, L.R.; Magaña, M.J.G. y Segura, C.J.C.F. 2008. Factores que afectan el porcentaje de gestación a 120 días posparto en vacas Cebú y cruzadas con europeo en Yucatán. Av lnv Agrop, 12: 45-56.

Aguilar, P.C.F. 2007. Energy balance and reproductive performance in grazing crossbreed cows in the tropics. PhD Thesis. University of Nottingham Sutton Bonington. Loughborough. Leicestershire. UK. 303 pp.

Archer, J.A.; Richardson, E.C.; Hera, R.M. and Arthur, P.F. 1999. Potential for selection to improve efficiency offeed use in beef cattle: a review. Austr J Agric Res, 50: 147-161.

Calegare, L.; Alencar, M.M.; Packer, I.U. and Lanna, D.P.D. 2007. Energy requirements and cow/calf efficiency of Nellore and Continental and British B. taurus $x$ Nellore Crosses. J Anim Sci, 85: 2413-2422.

Calegare, L.; Alencar, M.M.; Packer, I.U.; Leme, P.R.; Ferrell, C.L. and Lanna, D.P.D. 2009. Preweaning performance and body composition of calves from straightbred Nellore and Bos taurus $\times$ Nellore crosses. J Anim Sci, 87: 1814-1820.

Cárdenas, M.J.V.; Kú, V.J.C. and Magaña, M.J.G. 2010. Estimation of metabolizable energy requirements formaintenance and energetic efficiency of weight gain in Bos taurus and Bos indicus cows in tropical Mexico. J Anim Vet Adv, 9: 421-428.

Cundiff, L.V.; Bishop, M.D. and Johnson, R.K. 1993. Challenges and opportunities for integrating genetically modified animals into traditional animal breeding plans. J Anim Sci, 71: 20-25.

Davis, M.E.; Rutledge, J.J.; Cundiff, L.V. and Hauser, E.R. 1983a. Life cycle efficiency of beef production. I. efficiency ratios for progeny weaned. J Anim Sci 57: 832-851.

Davis, M.E.; Rutledge, J.J.; Cundiff, L.V. and Hauser, E.R. 1983b. Life cycle efficiency of beef production. II. Relationship of cow efficiency ratios for traits of the dam and progeny weaned. J Anim Sci, 57: 852-866.

Duch, G.J. 1988. La conformación territorial del estado de Yucatán: los componentes del medio físico. Universidad Autónoma Chapingo. Centro Regional de la Península de Yucatán. México. 427 pp.

Ferrell, C.L. and Jenkins, T.G. 1984a. A note on energy requirements for maintenance of lean and fat Angus, Hereford and Simmental cows. Anim Prod, 39: 305-309.

Ferrell, C.L. and Jenkins, T.G. 1984b. Energy utilization by mature, nonpregnant, nonlactating cows of differenttypes. JAnim Sci, 58: 234-243.

Ferrell, C.L. and Jenkins, T.G. 1985. Cow type and the nutritional environment: Nutritional aspects. J Anim Sci, 61: 725-741.

Ferrell, C.L. and Jenkins, T.G. 1998a. Body composition and energy utilization by steers of diverse genotypes fed a high-concentrate diet during the finishing period: I. Angus, Belgian Blue, Hereford, and Piedmontese sires. J Anim Sci, 76: 637-646.

Ferrell, C.L. and Jenkins, T.G. 1998b. Body composition and energy utilization by steers of diverse genotypes fed a high-concentrate diet during the finishing period: II. Angus, Boran, Brahman, Hereford and Tuli sires. J Anim Sci, 76: 647-657.

Fox, D.G.; Tedeschi, L.O. and Baker, M.J. 2002. Determining pot-weaning feed efficiency in beef cattle. Proc. Beef Improvement Federation. 34th Ann Met. Omaha, NE. USA., pp: 44-66.

Francisco, C.L.; Cooke, R.F.; Marques, R.S.; Mills, R.R. and Bohnert, D.W. 2012. Effects of temperament and acclimation to handling on feedlot performance of Bos taurus cattle originated from a rangeland-based cow-calf system. J Anim Sci, 90: 5067-5077.

Green, R.D.; Cundiff, L.V.; Dickerson, G.E. and Jenkins, T.G. 1991 a. Output/input differences among nonpregnant, lactating Bos indicusBos taurus and Bos taurus-Bos taurus F1 cross cows. J Anim Sci, 69: 3156-3166.

Green, R.D.; Cundiff, L.V. and Dickerson, G.E. 1991b. Life-cycle biological efficiency of Bos indicus $\times$ Bos taurus crossbred cow-calf production to weaning. J Anim Sci, 69: 3544-3563.

Jenkins, T.G., Cundiff, L.V. and Ferrell, C.L. 1991. Differences among breed crosses of cattle in the conversion of food energy to calf weight during the preweaning interval. J Anim Sci, 69: 2762-2769.

Jenkins, E.R. and Ferrell, D.C. 1992. Lactation characteristics of nine breeds of cattle fed various qualities of dietary energy. J Anim Sci, 70: 1652-1660.

Jenkins, E.R. and Ferrell, D.C. 2004. Preweaning efficiency for mature cows of breed crosses from tropically adapted Bos indicus and Bos taurus and unadapted Bos taurus breeds. J Anim Sci, 82: 1876-1881.

Johnson, D.E.; Ferrell, C.L. and Jenkins, T.G. 2003. The history of energetic efficiency research: where have we been and where are we going?. J Anim Sci, 81: E27-E38.

Magaña, J.G. and Segura, C.J.C. 2001. Estimates of breed and heterosis effects for some reproductive traits of Brown Swiss and Zebu-related breeds in South-eastern Mexico. Livest Res Rural Dev, 13: 5.

Magaña, J.G.; Parra, B.G.M.; Estrada, L.R.J.; Ku, V.J.C. y Sosa, F.C.F. 2009. Caracterización del recurso genético animal en el diseño de sistemas sustentables de producción bovina en el trópico. Trop Subtrop Agroecosyst, 10: 85-94. 
Martínez, V.G.; Borrayo, Z.A.; Montaño, B.M.; Bustamante, G.J.J.; Palacios, F.J.A.; Vega, M.V.E. y Ríos, U.A. 2012. Producción de leche de vacas Criollo, Guzerat y sus cruzas recíprocas F1 y su relación con el peso al destete de las crías. Rev Mex Cienc Pec, 3: 501-514.

McDowell, R.E., Wilk, J.C. and Talbott, C.W. 1996. Economic viability of crosses of Bos taurus and Bos indicus for dairying in warm climates. J Dairy Sci, 79: 1292-1303.

Moe, P.W. and Tyrrell, H.F. 1972. Metabolizable energy requirements of pregnant dairy cows. J Dairy Sci, 55: 480.

Mejía, B.G.T.; Magaña, M.J.G.; Segura, C.J.C.; Delgado, L.R. y Estrada, L.R.J. 2010. Comportamiento reproductivo y productivo de vacas Bos indicus, Bos staurus y sus cruces en un sistema de producción vaca:cría en Yucatán, México. Trop Subtrop Agroecosyst, 12: 281-301.

Montaño, B.M.; Nielsen, M.K. and Deutscher, G.H. 1990. Energy requirements for maintenance of crossbreed beef cattle with different genetic potential for milk. J Anim Sci, 68: 2279-2288.

Montaño, B.M. and Nielsen, M. 1990. Biological efficiency to weaning and to slaughter of crossbred beef cattle with differentgenetic potential for milk. J Anim Sci, 68: 2297-2309.

NRC. 2000. Nutrient requirements of beef cattle. Updated $7^{\text {th }}$ ed. National Academy Press. Washington, D.C.

Olson, T.A.; Van Dijk, A.; Koger, M.; Hargrove, D.D. and Franke, D.E. 1985. Additive and heterosis effects on preweaning traits, maternal ability and reproduction from crossing of the Angus and Brown Swiss breeds in Florida. J Anim Sci, 61: 1121-1131.

Plasse, D.; Fossi, H.; Hoogesteijn, R.; Verde, O.; Bastidas, P.; Rodríguez, C. y Rodríguez, R. 2000. Producción de vacas $F_{1}$ Bos taurus $x$ Brahman apareadas con toros Brahman y de vacas Brahman con toros $F_{1}$ Bos taurus $x$ Brahman versus Brahman. 1. Pesos al nacer, destete, 18 meses y peso final. Livest Res Rural Dev, 12 (4).

Rodríguez, Y. and Ponce de León, R. 2011. Milk yield characterization (from 1986 to 2007) in Cuban dairy Zebu cattle genotypes (3/4 Zebu: $1 / 4$ Holstein) and their cross breeds. Cuban J Agric Sci, 45: 231-236.

Rueda, B.L.; Blake, R.W.; Nicholson, C.F.; Fox, D.G.; Tedeschi, L.O.; Pell, A.N.; Fernández, E.M.C.; Valentín, J.F. and Carneiro, J.C. 2003. Production and economic potentials of cattle in pasture-based systems of the western Amazon region of Brasil. J Anim Sci, 81: 2923-2937. Sandelin, B.A.; Brown Jr, A.H.; Brown, M.A.; Johnson, Z.B.; Kellogg, D.W. and Stelzleni, A.M. 2002. Genotype $x$ environmental interaction for mature size and rate of maturing for Angus, Brahman, and reciprocal-cross cows grazing bermudagrass or endophyte infected fescue. J Anim Sci, 80: 3073-3076.

SAS. 2002. SAS/STAT User's Guide. SAS Institute. Cary, North Caroline. Solis, J.C.; Byers, F.M.; Schelling, G.T.; Long, C.R. and Greene, L.W. 1988. Maintenance requirements and energetic efficiency of cows of different breed types. J Anim Sci, 66: 764-773.

Steel, R.G.D. and Torrie, J.H. 1980. Principles and procedures of statistics: A biometrical approach (2nd Ed). McGraw-Hill Inc., New York.

Turner, J.W. 1980. Genetic and biological aspects of Zebu adaptability. J Anim Sci, 50: 1201-1205.

Yilma, Z.; Goijam, Y. and Çshumye, M. 2006. Milk production level and calf-rearing system affecting Boran, Ethiopian zebu cattle breed, cow-calf performance. Livest Res Rural Dev, 18 (71). 